\title{
Reply to the Letter to the Editor: Citation Analysis in Bariatric Surgery
}

\author{
Suhaib S. Ahmad ${ }^{1} \cdot$ Sandro Kohl $^{2}$ • Sami Ahmad ${ }^{3}$ • Ahmed R Ahmed ${ }^{4}$. \\ Sufian S. Ahmad ${ }^{2}$
}

Published online: 16 August 2015

(C) Springer Science+Business Media New York 2015

Reply to the letter to the editor,

Aminian et al. submitted a letter to the editor discussing our paper entitled "The hundred most cited articles in bariatric surgery" [1]. We thank the commentators for the scrutiny and appreciate their critical response.

The authors correctly noted in their letter the fact that a similar paper of their own was published in the Journal of Surgery for Obesity Related Disease in September 2014.

The authors of the letter compared the two lists of citation classics between both articles and identified 16 articles missing in the later published study, attributing this to the use of only two keywords.

After having carefully studied both articles, it is to be underlined that the authors of the letter, who we very much thank for the concern and additional review, are unfortunate in being mistaken in some of the conclusions drawn based on the simple comparison between the two lists undertaken; the methodology of the two studies notably differed. Less attention was drawn to the fact that the two studies were conducted using two completely different search databases. At this juncture, it would be helpful to provide a slight glimpse of the history of bibliometric science. Eugene Garfield, considered the father of scientometrics, established the Institute for Scientific Information (ISI) in the

Sufian S. Ahmad

sufiansamy@gmail.com

1 Buckingham Institute for Translational Medicine, University of Buckingham, Buckingham, UK

2 Department of Orthopaedic Surgery and Traumatology, Inselspital, University of Bern, Bern, Switzerland

3 Bariatric Surgery, Jordan Hospital, Amman, Jordan

4 Department of Bariatric and Metabolic Surgery, Imperial College London, London, UK 1960s, for the sake of providing a possibility of processing the huge flow of scientific information experienced at that time. Now the ISI, in the hands of Thomson Reuters, provide listings of journals by subject category alongside relevant indices based on the ground of citation indexing, which was a novel principle at the time of the founding of the institute. Journals are well assessed for eligibility before being included in the ISI lists, and citing sources of ISI-listed articles carefully identified based on set criteria. The scholarly indices published by ISI are those agreed on by the vast majority of journals, scientists, and institutions worldwide and are used widely for academic ratings and promotional purposes $[2,5-8]$. Nevertheless, in more recent years, more databases have appeared including scopus and google scholar, these cover up a wider range of journals, however, also contain non-peer-reviewed material which cautiously needs to be taken into consideration [4]; a citation is a citation, but is the value of a New England citation of Smithrins article the same as that of a regional non-indexed weekly? We do believe that the ISI database, at least for comparative reasons, needs to be part of scientometric studies until further developments in the field occur.

The best example could be made by having a closer look at table 1 in the letter of Aminian et al. There are two columns illustrating the number of citations of the 16 proposed missed articles: the left column with the citation count as of December 2013 retrieved from the Scopus database, from Aminians original study [3], and the right column with the ISI web of knowledge citation count as of March 2015. Nine of the sixteen studies (56\%) had fewer citations in the ISI web of knowledge database 15 months later.

Additionally, the inclusion criteria of studies, which were set prior to the search begin in our published study could have accounted for some of the observed differences, which also add to the methodological differences, not to neglect the time interval between the two articles. 
Metadata is a very vital part of any paper. It comprises the keywords, title, abstract, and the author details of any manuscript.

Keywords are vital, especially because of their frequent use in narrowing search results. Thus, it is of great significance to be very careful when choosing keywords, which should contain the most important words from the abstract and reflect the specificity of the article.

Many researchers do not pay much attention to the importance of keywords resulting in their articles being missed by subsequent papers.

The methodology of our study was reviewed by several experts prior to peer review including research experts in the field of bibliometrics, an emeritus editor in chief and also by further academic consultant surgeons. All agreed on an appropriate methodology, followed by a peer-review process with the involvement of multiple Obesity Surgery journal reviewers.

We do believe that the letter did highlight some of the methodical difficulties associated with bibliometric studies. Due to the dynamic nature of science, updates of bibliometric studies are necessary, and the author of the next bibliometric study in bariatric surgery will for sure encounter our current discussions.

We are pleased to end our reply by greatly thanking the commentators for listing some very valuable impacting articles and raising some issues of interest, not only to bariatric surgeon, but also to the academic society.

\section{References}

1. Ahmad SS, Ahmad SS, Kohl S, et al. The hundred most cited articles in bariatric surgery. Obes Surg. 2015;25(5):900-9. doi:10.1007/ s11695-014-1542-1.

2. Ahmad SS, Evangelopoulos DS, Abbasian M, et al. The hundred most-cited publications in orthopaedic knee research. J Bone Joint Surg. 2014;96(22):e190.

3. Aminian A, Daigle CR, Brethauer SA, et al. Citation classics: top 50 cited articles in bariatric and metabolic surgery. Surg Obes Relat Dis. 2014;10(5):898-905. doi:10.1016/j.soard.2013.12.021.

4. Falagas ME, Pitsouni EI, Malietzis GA, et al. Comparison of pubmed, scopus, web of science, and google scholar: strengths and weaknesses. FASEB J. 2008;22(2):338-42. doi:10.1096/fj.079492LSF.

5. Garfield E. 100 citation classics from the Journal of the American Medical Association. JAMA. 1987;257(1):52-9.

6. Garfield E. 'The application of citation indexing to journals management. Curr Contents. 1994;33:3-5.

7. Garfield E, Merton RK. Citation indexing: its theory and application in science, technology, and humanities, vol. 8. New York: Wiley; 1979.

8. Margolis J. Citation indexing and evaluation of scientific papers. Science. 1967;155(3767):1213-9. 